\title{
Automatic Segmentation of Remote Sensing Images on Water Bodies Based on Image Enhancement
}

\author{
Jie Zhang ${ }^{1,2}$, Minquan Feng ${ }^{1 *}$, Yu Wang ${ }^{1}$ \\ ${ }^{1}$ State Key Laboratory Base of Eco-Hydraulic Engineering in Arid Area, Xi'an University of Technology, Xi'an 710048, \\ China \\ ${ }^{2}$ Shanxi Conservancy Technical College, Yuncheng 044004, China
}

Corresponding Author Email: mqfeng@xaut.edu.cn

https://doi.org/10.18280/ts.370616

Received: 2 July 2020

Accepted: 6 October 2020

\section{Keywords:}

image enhancement, remote sensing image, water bodies, image segmentation, adaptive morphology

\begin{abstract}
By virtue of high-resolution remote sensing satellites, there is a possibility to analyze remote sensing images on water bodies through digital image processing (DIP). In many remote sensing images, however, the water bodies have similar gray values as other ground objects. To effectively distinguish water bodies from other ground objects in these images, this paper proposes a logarithmic enhancement method for remote sensing images on water bodies based on adaptive morphology. The proposed method can filter the noise of non-target area, and enhance the water body in the original image. On this basis, a morphology-based segmentation method was designed for remote sensing images on water bodies. Experimental results show that our method achieved a high segmentation accuracy, controlling the mean segmentation error at below $1.32 \%$.
\end{abstract}

\section{INTRODUCTION}

Remote sensing is a comprehensive technology that collects the electromagnetic waves of ground objects with remote sensors, and analyzes the waves to identify, monitor, or quantify the regions of interest (ROIs) $[1,2]$. Recently, the resolution of remote sensing satellites has been improved to the sub-meter range, laying technical basis for the management of land resources.

Under the effects of sensor features, weather, and illumination, noise and distortion may arise in the transmission, formation, and transform of remote sensing images [3], calling for effective processing of these images. In essence, the processing of remote sensing images is to segment, classify, or recognize the objects in the images through digital image processing (DIP). The DIP combines computer and mathematical methods to process and transform the original image to an image with higher utilization value.

This paper relies on the DIP to segment the remote sensing images on water bodies, and counts the area of each water body, providing the data for water resource management and environmental protection. Specifically, the original image was enhanced by a logarithmic method based on adaptive morphology, and then processed by a morphology-based segmentation method. Experimental results demonstrate the real-time performance and efficiency of our method over traditional manual measurement.

\section{LITERATURE REVIEW}

Surface water, mainly including lakes, rivers, and coastline extension areas, is an important part of water resources. The accurate segmentation of surface water is of great help to flood prevention, water resource assessment, and environmental protection. Therefore, various methods have been proposed to process and segment remote sensing images on water bodies.

\subsection{Segmentation based on spectral and texture features}

Liu et al. [4] put forward a river target detection algorithm based on multi-feature fusion: the original remote sensing image was meshed into grids; random forest training and classification were implemented based on the local entropy, texture features, spectral features, and color features of the grids; the detection results of machine learning were optimized through morphological operation.

Yuan and Sarma [5] proposed a river target detection method based on multiple features and wavelet support vector machine (SVM): the gray features of neighboring pixels were measured by mean value ratio with Gabor wavelet; the texture features were extracted through wavelet transform, and constructed into training samples; the normalized feature matrix was trained by wavelet SVM, and used to classify image pixels; the background noises like shadows and lakes were removed based on regional connectivity and shape features of rivers, leaving only the river parts.

\subsection{Object-oriented segmentation}

Wu et al. [6] analyzed the image feature space with various maps, e.g., land use map, topographic map, and water system map, segmented noises like shadows and vegetation by traditional method, and initially extracted the water areas; Then, the water areas were optimized through LBV (land, water body, vegetation) transform and object-oriented segmentation; Finally, the water areas were further refined through morphology operation and SVM supervision.

Wang et al. [7] presented an object-oriented method to extract aquaculture water areas: First, a multi-precision image 
segmentation method was designed to preliminarily segment the original image; Then, the spectral, texture, and shape features of the image were calculated, as well as the spatial relationship; Finally, the aquaculture water areas were extracted through multi-feature analysis.

\subsection{Segmentation based on Markov and generalized Gamma distribution [8]}

Biswas et al. [9] modeled the clutter in remote sensing images based on generalized Gamma distribution, and developed a coastline detection method based on level set segmentation, which detects coastlines in an accurate manner.

Drawing on Markov random field, Baumgartner et al. [10] proposed a segmentation method for multi-spectral remote sensing images: the eigenvectors of each frequency band were calculated, the feature parameters of each upper and lower frequency band were estimated, the local smoothing filtering was performed, and the eigenvectors were combined into the segmentation results.

Han et al. [11] segmented remote sensing images on rivers with the mixed active contour model of regional information fusion, and computed the cross entropy and variance of the gray values of the outer and inner regions of the contour curve, using the cross entropy active contour model, such that the curve gradually approached the target edge. This approach applies to the segmentation of remote sensing images on rivers with certain contrast.

\subsection{Adaptive morphology}

Morphological operation could easily change the edge and other attributes of the original image. Thus, it is important to realize adaptive morphological operation by setting up suitable structural elements. Many scholars [12-15] have successfully constructed morphological adaptive structural elements.

Teng et al. [16] proposed an HSI (hue-saturation-intensity) restoration method based on the structure information of color images obtained through adaptive morphological filtering and fusion. By this method, the adaptive structural elements of each pixel in the original image could be constructed, without any mixed noise, resulting in a good spatial structure.

Legaz-Aparicio [17] proposed an adaptive morphological close operation, which uses the inertia tensor to estimate the geometric features of the local structure of the image, and then constructs the structural elements by these geometric features. Sun et al. [18] proposed the concept of similarity weight by graph space, defined adaptive structural elements, and created an adaptive morphological operator.

\section{LOGARITHMIC ENHANCEMENT BASED ON ADAPTIVE MORPHOLOGY}

The enhancement of remote sensing images serves two purposes: improving the overall vision of the original image, and highlighting the targets in the ROIs. Image enhancement is critical to the effective utilization of remote sensing images on water bodies. These images often have low contrast, due to the presence of many non-water areas with complex shapes. The low contrast hinders the subsequent segmentation and analysis of water areas.

For the following reason, this paper constructs adaptive elliptical structural elements (ESEs) by linear structure tensor: an ESE, shaped between a straight line and a circle, can adapt to the target edge as much as possible, and maintain the spatial structure of water edge. Since the adaptive morphology can effectively filter out the noises outside the target, the authors designed a logarithmic enhancement method based on adaptive morphology, thereby enhancing the water bodies and suppressing the noises.

\subsection{Logarithmic enhancement method}

The common image enhancement methods are generally based on spatial domain or frequency domain. The spatial domain-based methods process image pixels directly, including histogram equalization, logarithmic transform, and spatial filtering.

Histogram equalization automatically adjusts image contrast through gray transform. The gray histogram of the original image is stretched nonlinearly to expand the dynamic range of gray values of image pixels, making them obey uniform distribution. Let $h(r)$ be the gray histogram of the original image, and $[0, S-1]$ be the range of gray values. Then, the total number of pixels of the original image can be expressed as:

$$
P_{N}=\int_{0}^{S-1} h(r) d r
$$

where, $r$ is the gray value of the original image.

The probability density function $p(r)$ and the probability distribution function $P(r)$ can be respectively defined as:

$$
\begin{gathered}
p(r)=h(r) / P_{N} \\
P(r)=1 / P_{N} \int_{0}^{S-1} h(r) d r
\end{gathered}
$$

Since the total number of pixels $P_{N}$ of the original image equals the number of pixels $P_{N}^{\prime}$ of the equalized image, we have

$$
h(t) d t=h(r) d r
$$

where, $s$ is the gray value of the equalized image; $h(t)$ is histogram of the equalized image.

The derivative of the histogram equalization transform $T(r)$ can be defined as:

$$
T^{\prime}(r)=d t / d r=h(t) / h(r)
$$

If $T^{\prime}(r)=\left(C / P_{N}\right) h(r)$, then $C$ is a constant, and $h(t)$ must also be a constant.

Therefore, the histogram equalization transform $T(r)$ can be defined as:

$$
T(r)=C / P_{N} \int_{0}^{S-1} h(r) d r=C P(r)
$$

The logarithmic curve is gentler than the straight line in an area with small pixel value, and steeper than the latter in an area with large pixel value. Thus, the nonlinear transform by logarithmic function can increase the gray value of dark areas and reduce that of bright areas. The logarithmic transform can be defined as: 


$$
g_{s}=\operatorname{clog}_{s c+1}(1+s c \times r)
$$

where, $c$ is a constant; $r$ is the gray value of the original image; $g_{s}$ is the gray value after logarithmic transform; $s c$ is the scale coefficient.

The above common methods for image enhancement feature are fast in calculation, and capable of highlighting target edges and suppressing noises. However, there are many non-target areas with complex surface features in the remote sensing images on water bodies. It is impossible for the common methods to filter out the noises and enhance the water bodies simultaneously. Thus, this paper proposes a logarithmic enhancement method for these images based on adaptive ESEs (Figure 1).

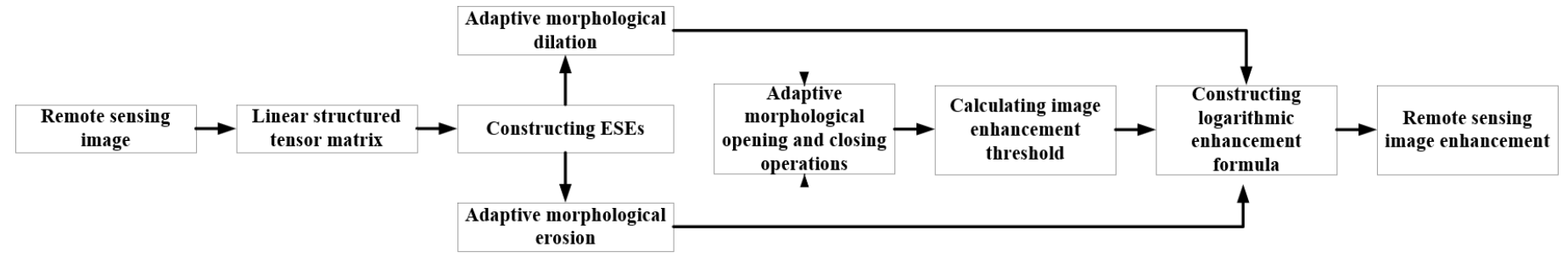

Figure 1. The flow chart of the logarithmic enhancement method based on adaptive ESEs

As shown in Figure 1, A linear structured tensor matrix was established for the original image, and used to construct the ESEs. Then, adaptive morphological dilation and erosion were defined, and adaptive morphological opening and closing operations were implemented to calculate the image enhancement threshold. Finally, the remote sensing image was enhanced through the logarithmic enhancement of dilation and erosion structures.

\subsection{Morphological operation of adaptive ESEs}

The fixed structural element could easily produce new artificial target, alter the position of target edges, destroy the boundary of the transition zone between the original targets, and lose track of small targets in morphological operation. Hence, the $\operatorname{ESE} E_{e s}(a, b, \varphi)$ was designed based on the linear structure tensor (Figure 2).

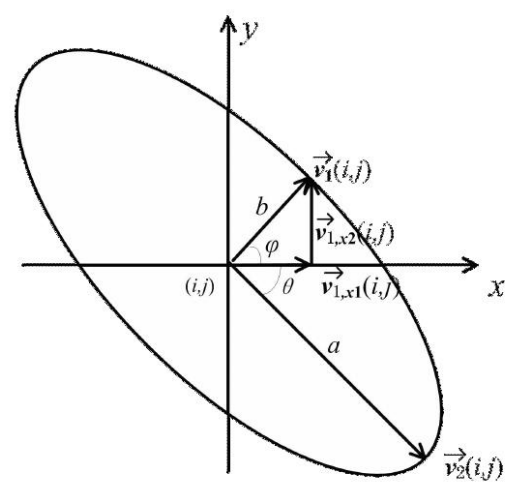

Figure 2. The ESE

Note: $a$ and $b$ are the major axis and minor axis semi-diameters of the ellipse, respectively; $\varphi$ is the angle between the minor axis semi-diameter and the $\mathrm{x}$ axis of the ellipse.

The adaptive morphological dilation and erosion follows roughly the same principle as classical morphology. The only difference is that the structural elements of adaptive morphological dilation and erosion can adapt their size and shape to the image, while those of classical morphology have fixed size and shape.

During adaptive morphological erosion, the shape and direction of the ESEs changed with the image features, and the minimum pixel values was taken in the ESEs. Contrary to the erosion, adaptive morphological dilation chose the maximum pixel values in the ESEs. The ESEs of the adaptive morphological erosion $E_{o}$ and dilation $D_{o}$ can be respectively defined as:

$$
\begin{aligned}
& E_{o}(g)=\bigwedge_{(i, j) \in E} g(i, j) \quad \forall E \in g \\
& D_{o}(g)=\bigvee_{(i, j) \in E} g(i, j) \quad \forall E \in g
\end{aligned}
$$

where, $E$ is an ESE; $g$ is the pixel value of the original image; $(i, j)$ is a pixel; $\Lambda$ and $\vee$ are the minimization and maximization of the pixel value in domain $E$, respectively.

The opening operation $\theta$ and the closing operation $\rho$ are the combination of erosion $E_{o}$ and dilation $D_{o}$. Morphological adaptive opening and closing operations can be respectively defined as:

$$
\begin{gathered}
\theta(g)=(g \circ E) \\
\rho(g)=(g \circ E)
\end{gathered}
$$

where, $\circ$ and $\odot$ are indicators of morphological opening and closing operations, respectively.

In remote sensing images of water bodies, water, trees and building shadows have similar gray values. Under force majeure, these images tend to have a low contrast, contain lots of noises, and face many adverse factors. These have a major impact on the quality of water body segmentation. The noises, such as the shadows of trees and buildings, could be eliminated through adaptive morphological operation, thereby retaining the edges of water bodies.

The threshold $\tau=(\theta+\rho) / 2$ of image enhancement was calculated by opening and closing operations. Then, the pixel value of the original image $g$ was compared with the threshold $\tau$. The original image was enhanced through erosion, if the pixel value was smaller than the threshold, and enhanced through dilation, if otherwise. Therefore, the logarithmic enhancement formula can be defined as:

$$
\xi(g)=\left\{\begin{array}{lr}
k \lg (g+1)+\theta(g) & g<\tau \\
k \lg (g+1)+\rho(g) & \text { otherwise }
\end{array}\right.
$$

where, $k=(255-\tau) / \lg 256$.

Compared with the traditional logarithmic enhancement (7), the proposed method introduces the idea of self-adaptation. 
The constant $c$ in formula (7) was replaced by the parameter $k$, which is related to the opening and closing operations of adaptive ESEs. Based on the results of adaptive dilation and erosion, the water bodies could be enhanced without sacrificing the edges of water bodies, while eliminating the noises outside the water bodies.

\section{ESES-BASED IMAGE SEGMENTATION}

Image segmentation aims to splits an image into multiple regions with certain features, and extract the ROIs from them. The popular image segmentation methods are grounded on threshold, edge, or region.

Threshold-based segmentation uses one or several thresholds to divide the gray histogram of an image into one or several classes. The pixels in the same gray range are treated as belonging in the same target region. The threshold segmentation can be defined as:

$$
g^{\prime}(x, y)= \begin{cases}1 & g(x, y) \geq T \\ 0 & g(x, y)<T\end{cases}
$$

where, $T$ is the threshold; $g^{\prime}(x, y)$ is a segmented image; $g(x, y)$ is the original image.

The threshold could be selected excellently by maximizing interclass variance. Let $s \times t$ be the number of pixels in the original image $g(x, y), T$ be the initial threshold, $n_{1}$ be the number of original image pixels whose value is smaller than the threshold, and $n_{2}$ be the number of original image pixels whose value is greater than the threshold. Then, the interclass variance can be expressed as:

$$
v_{g}=w_{0}\left(u_{0}-u\right)^{2}+w_{1}\left(u_{1}-u\right)^{2}
$$

where, $u$ is the mean gray value of original image; $w_{0}$ is the proportion of the number of original image pixels whose value is smaller than the threshold; $w_{1}$ is the proportion of the number of original image pixels whose value is greater than the threshold; $u_{1}$ is the mean gray value of original image pixels whose value is greater than the threshold $T$ :

$$
\begin{gathered}
w_{0}=n_{1} / s \times t \\
w_{1}=n_{2} / s \times t \\
n_{1}+n_{2}=s \times t \\
u=w_{0} u_{0}+w_{1} u_{1}
\end{gathered}
$$

The gray value of each pixel in the original image was compared with the initial threshold $T$. The best threshold was determined as the $T$ value that maximizes the interclass variance, i.e., the difference between foreground and background.

Edge-based segmentation often adopts the Laplacian operator. Laplacian operator is a second-order differential operator. It is an isotropic operator invariant to rotation and sensitive to isolated pixels. As a result, the operator is only suitable for image edge detection with no or very few noises. The Laplacian operator can be defined as:

$$
\nabla^{2} g=\frac{\partial^{2} y}{\partial x^{2}}+\frac{\partial^{2} g}{\partial y^{2}}
$$

The original image $g(x, y)$ can be written in discrete form as:

$$
\begin{aligned}
& \nabla^{2} g(x, y)=g(x+1, y)+g(x-1, y) \\
& +g(x, y+1)+g(x, y-1)-4 g(x, y)
\end{aligned}
$$

The Laplacian operator can determine the mutation of pixel value, using the zero-crossing point between the positive and negative peaks of quadratic differential. This is very suitable for detecting the image region of isolated point and line, or the combination of point and line.

In general, Laplacian operator needs to be used with a lowpass filter. This leads to the Laplacian of Gaussian (LoG) operator: the original image is smoothed by Gaussian filter, and then processed by the Laplacian operator to detect the edges. The LoG operator can be defined as:

$$
\begin{array}{r}
\nabla^{2} g(x, y)=1 / \pi \delta^{2}\left(x^{2}+y^{2} / 2 \delta^{2}\right. \\
-1) e^{-\left(x^{2}+y^{2}\right) / 2 \delta^{2}}
\end{array}
$$

where, $\delta$ is the standard deviation.

Region-based segmentation extracts interested objects by segmenting regions with different features from the original image. The commonly used approaches include region growing, and region splitting and merging.

Region growing selects a series of seed points with different regional features, merges the qualified pixels into the seed point neighborhoods, repeats the two steps iteratively until no qualified pixels could be found. Region growing can segment complex images without prior knowledge. However, the iterative algorithm consumes a long time, and fails to eliminate the noises, which may cause over-segmentation or leave holes in the target region. Thus, it is particularly important to determine high-quality seed points and growth criteria.

Region splitting and merging first splits the original image, and then merges the segments to achieve the effect of image segmentation. Splitting can be regarded as the inverse process of region growing, and merging as the region growing of a single pixel. Compared with region growing, region splitting and merging involve more splitting steps, and achieve better segmentation effect on complex images. Of course, the superior performance is realized at the expense of complex operations and heavy computing load. Besides, the splitting process often destroys the image edges. The key of region splitting and merging lies in the design of splitting and merging criteria. Proper criteria are the premise of accurate target segmentation.

The above three traditional methods face different problems in handling remote sensing images on water bodies, owing to the complex and noisy features. Threshold-based segmentation requires the selection of the best threshold. Despite its simplicity and time efficiency, this approach has a poor anti-noise performance and a high false segmentation rate In addition to these problem, edge-based detection might overlook edge changes and small targets. Region-based segmentation could falsely segment or miss the targets with uneven gray distribution.

This paper adopts the morphological operation of adaptive ESEs to eliminate the influence of dark detail noises on water bodies, and prevent the overstretching of the water bodies. On this basis, the water bodies were segmented by gray slice. The workflow of the ESEs-based image segmentation is shown in Figure 3. 


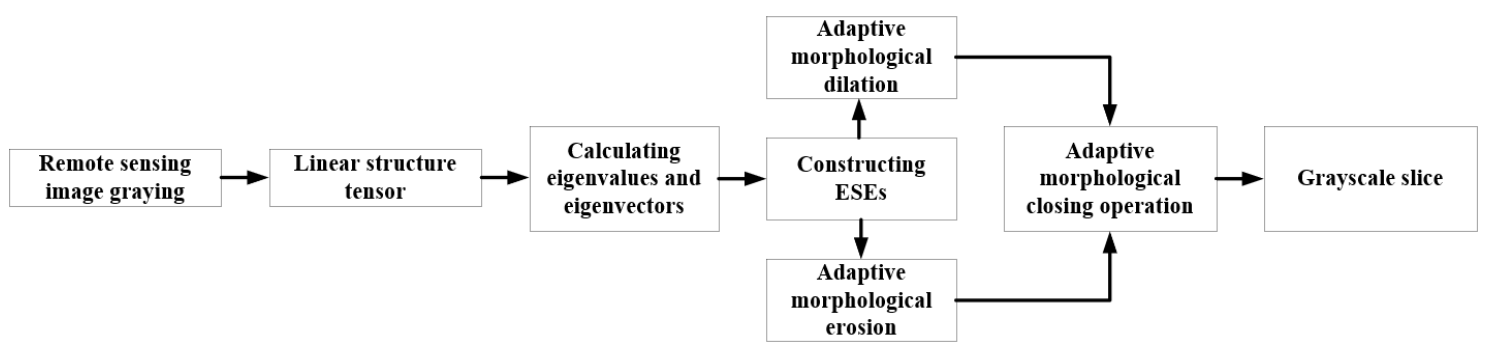

Figure 3. The flow chart of image segmentation based on adaptive ESEs

Gray slice mainly targets binary image and gray image. Since binary image has only two gray values, the gray slice of binary image directly sets the background to 0 or 1 , and the foreground to 1 or 0 . Because the gray range of gray image is $0-255$, the ROIs usually fall in a small gray range. Taking the target area as foreground and non-target area as background, the gray slice method was adopted to extract the gray value of the foreground.

Firstly, the gray histogram of the original image was calculated. Then, the target gray value area was determined by the gray change law of the histogram. Finally, the gray value of non-target area was set to 0 or 255 , and the gray value of the target area was retained or set to 255 or 0 corresponding. If the image contains multiple gray target areas, the gray slice could use multiple gray parameters to improve the segmentation and extraction effect. The grayscale slice can be defined as:

$$
h_{g}=\left\{\begin{array}{lr}
255 & 0<g(x, y) \leq a \\
0 & a<g(x, y) \leq b \\
255 & b<g(x, y) \leq 255
\end{array}\right.
$$

where, $a$ and $b$ are the foreground and background gray parameters of the original image, respectively; $h_{g}$ is the image histogram after grayscale slice.

The grayscale slice can be represented by a coordinate graph as Figure 4.

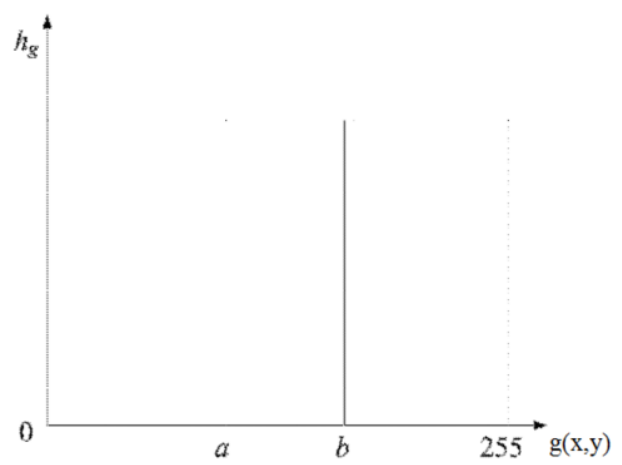

Figure 4. The gray slice function

\section{EXPERIMENT AND RESULTS ANALYSIS}

MATLAB experiments were conducted to verify the effects of the proposed logarithmic enhancement method on remote sensing images of water bodies in terms of edge preservation, denoising, and water body enhancement. The experimental results of different image enhancement methods are shown in Figure 5.

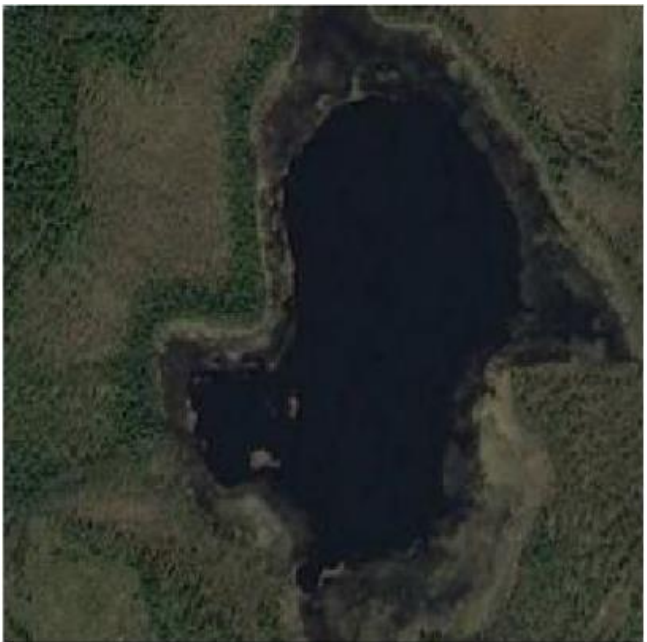

(a) Original image

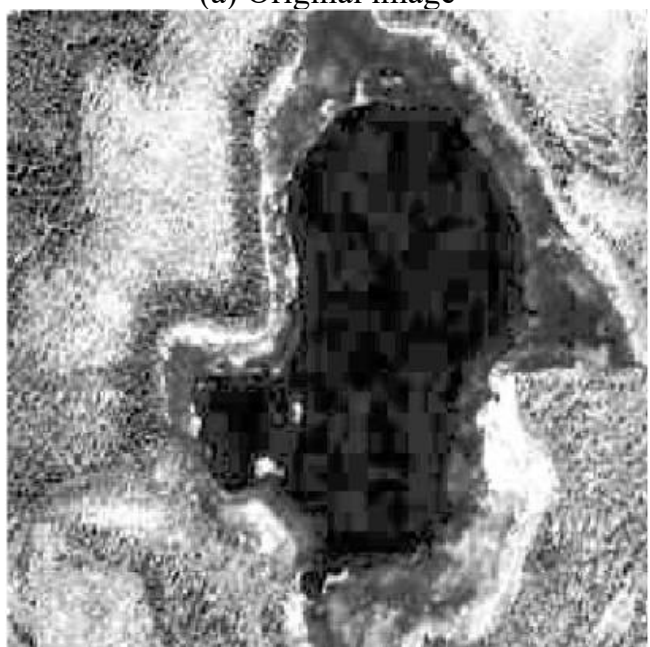

(b) Image after histogram equalization

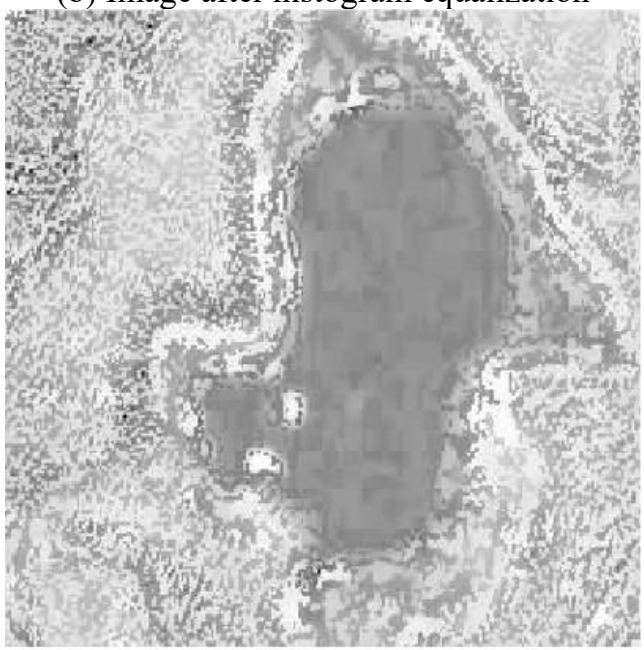

(c) Image after logarithmic enhancement based on circular structural elements 


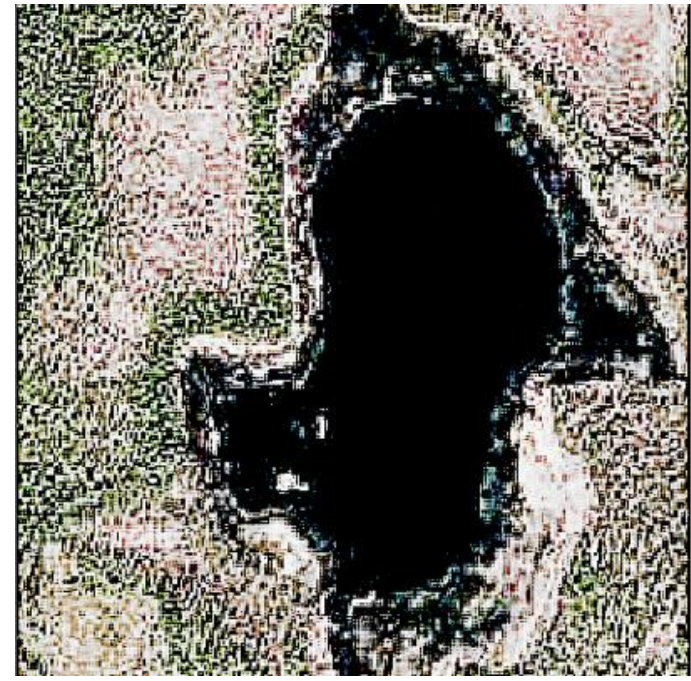

(d) Image after the proposed logarithmic enhancement method

Figure 5. The experimental results on different image enhancement methods

As shown in Figure 5, the pixels of the water body were not smooth enough in the image output by histogram equalization; the logarithmic enhancement based on circular structural elements amplified the noise area in the water body, due to the single shape of these elements; the lack of shape diversity changes the edge contour of the small noise in the water body, and distorts the edge of the water body. By contrast, our method eliminated the external noises, highlighted the water body, and preserved the edges of the water body excellently.

Next, the performance of our method were measured by contrast and information entropy [19]. Table 1 compares the contrast and information entropy of the image enhanced by different methods. It can be seen that our method achieved the experimental purpose well.

Finally, the number of pixels in the image segmented by each method was counted to compute the image segmentation error $E_{s}$ :

$$
E_{S}=\left|S N-S N_{0}\right| / S N_{0}
$$

Table 1. The contrast and information entropy of the image enhanced by different methods

\begin{tabular}{cccc}
\hline & $\begin{array}{c}\text { Histogram } \\
\text { equalization }\end{array}$ & $\begin{array}{c}\text { Logarithmic } \\
\text { enhancement based } \\
\text { on circular } \\
\text { structural elements }\end{array}$ & $\begin{array}{c}\text { Our } \\
\text { method }\end{array}$ \\
\hline $\begin{array}{c}\text { Contrast } \\
\text { Information } \\
\text { entropy }\end{array}$ & 718.62 & 618.57 & 112.68 \\
\hline
\end{tabular}

Table 2. The image segmentation errors of different methods

\begin{tabular}{cccc}
\hline $\begin{array}{c}\text { Logarithmic } \\
\text { enhancement } \\
\text { based on } \\
\text { circular } \\
\text { structural } \\
\text { element }\end{array}$ & $\begin{array}{c}\text { Logarithmic } \\
\text { enhancement } \\
\text { based on angle } \\
\text { adaptive linear } \\
\text { structural } \\
\text { elements }\end{array}$ & $\begin{array}{c}\text { Multi-point } \\
\text { region } \\
\text { growing } \\
\text { based on } \\
\text { morphologi } \\
\text { cal filtering }\end{array}$ & $\begin{array}{c}\text { Our } \\
\text { method }\end{array}$ \\
\hline 5.66 & 3.46 & 5.32 & 1.17 \\
\hline
\end{tabular}

where, $S N$ is the number of pixels of the segmented image; $S N_{0}$ is the number of pixels of manually segmented image.
Then, the image segmentation errors of different methods, including but not limited to logarithmic enhancement based on circular structural elements, that based on angle adaptive linear structural elements, multi-point region growing based on morphological filtering, are compared in Table 2. The mean segmentation error of our method was less than $1.32 \%$, smaller than that of any other method.

\section{CONCLUSIONS}

This paper introduces the morphological operation of adaptive ESEs to enhance the remote sensing images on water bodies. Experimental results show that, compared with the traditional method for adaptive morphological enhancement, our method filtered out the noises outside the target, enhanced the water body, and preserved the position and shape of the target edges. In addition, the authors developed an image segmentation algorithm for the said images based on the selfdesigned adaptive morphological ESEs, and the adaptive morphological closing operation. The proposed method eliminates the influence of dark detail noise on the water body, and prevents the over-stretching of water body edges, thereby accurately maintaining the edges of the water body.

\section{ACKNOWLEDGEMENT}

This work was supported by a General Financial Grant from the National Natural Science Foundation of China (Grant No.: 51679191) and the project funded by Research and Extension of Water Conservancy Science and Technology in Shanxi Province (Grant No.: 201625).

\section{REFERENCES}

[1] Ding, P., Zhang, Y., Jia, P., Chang, X.L. (2019). A comparison: different DCNN models for intelligent object detection in remote sensing images. Neural Processing Letters, 49(3): 1369-1379. https://doi.org/10.1007/s11063-018-9878-5

[2] Zeng, Y., Ning, Z., Liu, P., Luo, P., Zhang, Y., He, G. (2020). A mosaic method for multi-temporal data registration by using convolutional neural networks for forestry remote sensing applications. Computing, 102(3): 795-811. https://doi.org/10.1007/s00607-019-00716-5

[3] Chen, C., Li, W., Gao, L., Li, H., Plaza, J. (2018). Special issue on advances in real-time image processing for remote sensing. Journal of Real-Time Image Processing, 15(3): 435-438. https://doi.org/10.1007/s11554-0180831-7

[4] Liu, G., Kurban, A., Duan, H., Halik, U., Ablekim, A., Zhang, L. (2014). Desert riparian forest colonization in the lower reaches of Tarim River based on remote sensing analysis. Environmental Earth Sciences, 71(10): 4579-4589. https://doi.org/10.1007/s12665-013-2850-9

[5] Yuan, X., Sarma, V. (2010). Automatic urban waterbody detection and segmentation from sparse ALSM data via spatially constrained model-driven clustering. IEEE Geoscience and Remote Sensing Letters, 8(1): 7377. https://doi.org/10.1109/LGRS.2010.2051533

[6] Wu, Y.Q., Li, H.J., Song, Y. (2015). Target detection algorithm for rivers in SAR images based on multifeatures and WSVM. Systems Engineering and 
Electronics,

37(6):

$1288-1293$

https://doi.org/10.3969/j.issn.1001-506X.2015.06.10

[7] Wang, F., Xia, L., Chen, Z., Cui, W., Liu, Z., Pan, C. (2018). Remote sensing identification of coastal zone mariculture modes based on association-rules objectoriented method. Transactions of the Chinese Society of Agricultural Engineering, 34(12): 210-217. https://doi.org/10.11975/j.issn.1002-6819.2018.12.025

[8] Qiao, X., Yang, F., Zheng, J. (2019). Ground penetrating radar weak signals denoising via semi-soft threshold empirical wavelet transform. Ingénierie des Systèmes d'Information, 24(2):
https://doi.org/10.18280/isi.240213

[9] Biswas, B., Sen, B.K., Choudhuri, R. (2015). Remote sensing image fusion using PCNN model parameter estimation by Gamma distribution in shearlet domain. Procedia Computer Science, 70: 304-310. https://doi.org/10.1016/j.procs.2015.10.098

[10] Baumgartner, J., Gimenez, J., Scavuzzo, M., Pucheta, J. (2015). A new approach to segmentation of multispectral remote sensing images based on MRF. IEEE Geoscience and Remote Sensing Letters, 12(8): 1720-1724. https://doi.org/10.1109/LGRS.2015.2421736

[11] Han, B., Wu, Y., Song, Y. (2017). A novel active contour model based on median absolute deviation for remote sensing river image segmentation. Computers \& Electrical Engineering, 62: 209-223. https://doi.org/10.1016/j.compeleceng.2017.04.005

[12] Fan, Y., Shen, D., Gur, R.C., Gur, R.E., Davatzikos, C. (2006). COMPARE: classification of morphological patterns using adaptive regional elements. IEEE Transactions on Medical Imaging, 26(1): 93-105. https://doi.org/10.1109/TMI.2006.886812

[13] Bobin, J., Starck, J.L., Fadili, J.M., Moudden, Y., Donoho, D.L. (2007). Morphological component analysis: An adaptive thresholding strategy. IEEE Transactions on Image Processing, 16(11): 2675-2681. https://doi.org/10.1109/TIP.2007.907073

[14] Tripathy, D.P., Harish, Y.N. (2018). Prevention of illegal transportation using ANPR and biometric fingerprint in mining industry, Mathematical Modelling of Engineering Problems, 5(3): 249-255. https://doi.org/10.18280/mmep.050318

[15] Welfer, D., Scharcanski, J., Kitamura, C.M., Dal Pizzol, M.M., Ludwig, L.W., Marinho, D.R. (2010). Segmentation of the optic disk in color eye fundus images using an adaptive morphological approach. Computers in Biology and Medicine, 40(2): 124-137. https://doi.org/10.1016/j.compbiomed.2009.11.009

[16] Teng, Y., Zhang, Y., Chen, Y., Ti, C. (2015). Adaptive morphological filtering method for structural fusion restoration of hyperspectral images. IEEE Journal of Selected Topics in Applied Earth Observations and Remote Sensing, 9(2): 655-667. https://doi.org/10.1109/JSTARS.2015.2468593

[17] Legaz-Aparicio, Á. G., Verdu-Monedero, R., Angulo, J. (2018). Adaptive morphological filters based on a multiple orientation vector field dependent on image local features. Journal of Computational and Applied Mathematics, 330 965-981. https://doi.org/10.1016/j.cam.2017.05.001

[18] Sun, G., Bin, S., Jiang, M., Cao, N., Zheng, Z., Zhao, H., Wang, D., Xu, L. (2019). Research on public opinion propagation model in social network based on blockchain. Computers Materials \& Continua, 60(3):, 1015-1027. https://doi.org/10.32604/cmc.2019.05644

[19] Al-Ameen, Z. (2020). Satellite image enhancement using an ameliorated balance contrast enhancement technique. Traitement du Signal, 37(2): 245-254. https://doi.org/10.18280/ts.370210 